\title{
Endoscopic transforaminal lumbar interbody fusion in the treatment of degenerative lumbar spondylolisthesis
}

Denglu Yan ( $\square$ yandenglu@163.com )

First People's Hospital of Zhaoqing https://orcid.org/0000-0001-6503-5077

\section{Zaiheng Zhang}

Shenzhen Baoan People's Hospital

\section{Zhi Zhang}

Third Hospital of Guangzhou Medical University

Research article

Keywords: Lumbar, Spondylolisthesis, Endoscopic, Discectomy, Fusion

Posted Date: October 29th, 2019

DOI: https://doi.org/10.21203/rs.2.16488/v1

License: (1) (i) This work is licensed under a Creative Commons Attribution 4.0 International License.

Read Full License 


\section{Abstract}

Background There were no studies in literature of lumbar spondylolisthesis treated by endoscopic surgery. The purpose of this study was to evaluate the efficacy of endoscopic transforaminal lumbar interbody fusion (ELIF) in the treatment of degenerative lumbar spondylolisthesis by compare to the standard transforaminal lumbar interbody fusion (TLIF).

Methods A total of 93 patients with lumbar spondylolisthesis who had surgery from February 2017 to January 2018 were categorized into different groups depending on the procedure by ELIF or TLIF. The ELIF and TLIF procedures was performed, and the clinical outcomes of blood loss, operation times, hospital stay days, pain index, ODI score, the spondylolisthesis rate and reduction rate, and the disk height and intervertebral foramen height were recorded.

Results There was no intraoperative death in this series. In ends 86 cases had follow-up at least one year and 7 cases lost, and the follow-up rate and followed time were no difference between two groups $(P>$ 0.05). The operational time was longer in ELIF than TLIF $(P<0.01)$. The hospital days and blood loss were significant less in endoscopic group than TLIF $(P<0.01)$. The pain index and ODI score, the spondylolisthesis rate and reduction rate, and the disk height and intervertebral foramen height were better than preoperational $(P<0.01)$, and there were no difference between two groups $(P>0.05)$. All patients achieved spinal fusion with no cases of cage extrusion, and no infection, and no dural tear of cerebrospinal fluid leakage complication. There were one case of radiculitis (man) at endoscopic group. CT-myelogram revealed the radiculitis patients had normal radiologic findings, and the patient was recovered by neurotrophy drugs and functional exercises after 3 months.

Conclusions Endoscopic lumbar decompression and interbody fusion procedures was effective and safe measure in the treatment of the lumbar spondylolisthesis. Compare to open interbody fusion techniques, endoscopic lumbar interbody fusion was minimally invasive surgery with less bold loss and earlier postoperative recovery.

\section{Introduction}

With the development of minimally invasive endoscopic methods in spine surgery, the current trend of evolution lumbar spinal surgery has been toward endoscopic discectomy.[1-4] TLIF is a well-established technique for decompression and fusion by exposure of kambin triangle to access the disc space.

Compared to posterolateral fusion which uses a midline exposure and to decompression and fusion, TLIF has the advantages of minimally invasive by just a unilateral facetectomy.[5] However, the procedure for exposure of Kambin triangle need to unilateral facetectomy in TLIF, that damage the normal posterior structure of spine. Through the Kambin triangle, transforaminal endoscopic lumbar spine surgery has become population for its advantages and matured with time.[6-9] Transforaminal endoscopic lumbar surgery has become attractive procedures for lumbar spine because it not only don't damage the spinal structures but also safe procedures with less trauma and faster recovery.[10,11] 
Endoscopic lumbar interbody fusion (ELIF) is a minimally invasive for decompression and interbody fusion through the Kambin triangle.[10,12,13] Kambin triangle is an anatomic safe corridor to the intervertebral disc space bounded medially by the traversing nerve root, laterally by the exiting nerve root, and caudally by the pedicle. For TLIF, exposure of Kambin triangle is accomplished by facetectomy, the inferior articular process is removed, and the par is maintained to protect the dorsal root ganglion on cage insertion. Although the ELIF performed the decompression and interbody fusion through the Kambin as TLIF, it preserved the facet joint intact by the endoscopic techniques. The pedicle screw insertion in ELIF was also a minimally invasive by percutaneous techniques, it preserved the musculotendinous attachments and the integrity of lumbar fascia.[14]

Mandy studies comparing traditional TLIF to PLIF showed equal or better results with the transforaminal approach. Sufficient decompression with no retraction on the nerve ganglion, less postoperative pain and earlier functional recovery were the major advantages of the transforaminal approach.[5,15] With lumbar spine techniques development, the procedures of nerve decompression and interbody fusion was completed with maintain the facet intact, pedicle screw was placement by minimally invasive percutaneous techniques with preservation of musculotendinous attachments, ELIF true minimally invasive surgery has less blood loss, earlier postoperative recovery, and decreased rates of infection in comparison to open surgery.[16-18,14,19] The purpose of this study was to evaluate the efficacy of endoscopic transforaminal lumbar interbody fusion in the treatment of degenerative lumbar spondylolisthesis by compare to the standard transforaminal lumbar interbody fusion in our clinical.

\section{Materials And Methods}

From February 2016 to January 2018, a total of 93 consecutive patients of degenerative spondylolisthesis (grade I-II) were had ELIF and TLIF because of back and leg pain. Ninety-three patients initially fulfilled the study criteria, and seven patients were lost to follow-up. Of the remaining 86 patients available for analysis, 42 cases had ELIF (group 1, included 20 men and 22 women with an average age of $51.73 \pm 5.48$ years), and 44 patients had TLIF (group 2, included 23 men and 21 women with an average age of $52.16 \pm 5.39$ years). The inclusion criteria were adult degenerative spondylolisthesis (grades I-II) which only one or two level fusion (L3/4, L4/5, or couple levels). Exclusion criteria included pathologic conditions of the lumbar spine (trauma, tumor, or infection). The two groups had similar age and sex distribution, level of pain, and the pain history (Table 1).

Table 1 Patients general data (Means \pm SD)

\begin{tabular}{llllllll}
\hline Group & \multicolumn{2}{l}{ Gender } & \multicolumn{2}{l}{ Older } & Level & & History \\
& Male & Female & (years) & L3/4 & L4/5 & L3/4,4/5 & (years) \\
\hline $\operatorname{ELIF(42)}$ & 20 & 22 & $51.73 \pm 5.48$ & 11 & 19 & 12 & $3.16 \pm 1.52$ \\
\hline $\operatorname{TLIF}(44)$ & 23 & 21 & $52.16 \pm 5.39$ & 10 & 21 & 13 & $3.28 \pm 1.37$
\end{tabular}

There was no significant difference between two groups $(P>0.05)$ 


\section{Surgical procedures}

Under general anesthesia, all patients had nerve decompression, disc removed, bone graft and cage which packed with bone graft were inserted in the interbody space, and pedicle screw instrumentation was used. The TLIF procedure was performed in the standard fashion as reported in previous studies, and the ELIF procedures was performed under C-arm image step by step.[13,20,19] First, endoscopic discectomy and nerve decompression. After nerve decompression accomplished, the working tube for cage insertion was placed in the disc space, and rechecked by the endoscopic to ensure nerve and dual was safe and working tube was on the good position for subtotal discectomy and endplate preparation. Second, subtotal discectomy and endplate preparation was accomplished using a combination of straight and angled curettes and rasps.

Figure 1 Female patients of 39 years old had the procedures of ELIF for the spondylolisthesis of L4/5.

Then bone graft and expandable cage which packed with bone graft were inserted in the disc space. The expandable cage are inserted at a contracted height to allow safer entry into the disc space with less chance for impingement of the traversing and exiting nerve roots. The cage is then expanded in the interbody space to restore disc height and obtain interbody cage purchase. Maintaining the pars protects the exiting nerve root and dorsal root ganglion during cage insertion. Third, percutaneous pedicle screw were placement and compression be performed. Thorough release of the disc space and restoration of disc height aids in reduction of spondylolisthesis. If reduction of a spondylolisthesis is still desired, the rods can be fixed into the caudal vertebrae, and the pedicle screws from the cranial vertebra can be reduced to the rod. Fluoroscopic guidance should be used to ensure thorough discectomy and endplate preparation, bone graft and cage insertion, and percutaneous pedicle screw placement. Brace support was recommended for 4-6 weeks after surgery.

Figure 2 Male patients of 42 years old had the procedures of ELIF for the spondylolisthesis of L3/4 and L4/5

\section{Critical of clinical outcomes}

Before surgery and at the one year follow-up, operation times, blood loss, hospital stays, pain (Visual Analog Scale, VAS), functional disability (Oswestry Disability Index, ODI), and Mac Nab criteria were quantified in follow-up. All patients had preoperative and post-operative plain radiographs, computed tomography (CT) scans, and magnetic resonance (MR) images. The focus was to evaluate five radiographic characteristics at follow-up: (1) percentage of slip and percentage of reduction, (2) height of

disk space and intervertebral foramen, (3) cage position, and (4) fusion rate. Bone fusion was determined on the criteria of continuity of trabecular pattern, and the fusion rate assessed using CT-scan reconstruction. 


\section{Statistical analysis}

All measurements were performed by a single observer and are expressed as means \pm SD. Using the SPSS 17.0 statistics software, classic t-test and chi-square test were performed.

\section{Results}

There was no intraoperative death in this series. In ends 86 cases had follow-up at least one year and 7 cases lost, and the follow-up rate was 93.3\% (42/45) in the ELIF group and 91.7\% (44/48) in the TLIF group. The followed time from 14 to 24 months (average 18 months), and average $18.42 \pm 1.13$ months on ELIF and $18.57 \pm 1.24$ months on TLIF $(P>0.05)$.

The operational time was longer in ELIF than TLIF (138.62 \pm 30.54 minutes versus $87.84 \pm 20.73$ minutes, $\mathrm{P}<0.01)$.The hospital days were significant less in ELIF group than TLIF (4.12 \pm 1.64 days versus $9.36 \pm 1.57$ days, $P<0.01)$. The average blood loss were significant less in endoscopic group than TLIF $(115.72 \pm 20.83 \mathrm{~mL}$ versus $286.71 \pm 50.68 \mathrm{~mL}, \mathrm{P}<0.01)$.

Table 2 Follow up time and operation date of patients (Means \pm SD)

\begin{tabular}{|c|c|c|c|c|c|}
\hline Group & Follow up \# & & Opreationgal time ${ }^{*}$ & Hospital days ${ }^{*}$ & Blood loss ${ }^{*}$ \\
\hline & Rate & time & (Minutes) & (Days) & $(\mathrm{mL})$ \\
\hline ELIF & $93.3 \%(42 / 45)$ & $18.42 \pm 1.13$ & $138.62 \pm 30.54$ & $4.12 \pm 1.64$ & $115.72 \pm 20.83$ \\
\hline TLIF & $91.7 \%(44 / 48)$ & $18.57 \pm 1.24$ & $87.84 \pm 20.73$ & $9.36 \pm 1.57$ & $286.71 \pm 50.68$ \\
\hline
\end{tabular}

Note: ${ }^{*}$, significant difference $(P<0.01) .{ }^{\#}$, no significant difference $(P>0.05)$

The pain index of VAS improved from $7.56 \pm 1.13$ to $1.84 \pm 0.89(P<0.01)$ in ELIF patients and improved from $7.82 \pm 1.09$ to $1.45 \pm 0.91(P<0.01)$ in TLIF patients. The ODI improved from $72.48 \pm 15.63$ preoperatively to $21.37 \pm 11.82(\mathrm{P}<0.01)$ postoperatively in ELIF, and from $75.81 \pm 18.49$ preoperatively to $24.65 \pm 17.36(\mathrm{P}<0.01)$ postoperatively in ELIF. On the basis of Mac Nab criteria, there were 27 cases of excellent, 10 cases of good, 5 cases of general, and 0 cases of poor in ELIF group; there were 28 cases of excellent, 11 case of good, 5 case of general, and 0 cases of poor in TLIF group. The Mac Nab criteria in all patients was $84.1 \%$ of good or excellent (83.5\% in PLIF and $84.6 \%$ in TLIF, P > 0.05 ).

The average of spondylolisthesis was $30.18 \pm 7.24 \%$ in preoperatively and $5.37 \pm 2.14 \%$ in postoperatively in ELIF $(P<0.01)$, and from $31.46 \pm 8.31 \%$ to $5.44 \pm 2.76 \%$ in TLIF $(P<0.01)$. The average of reduction rate was $75.23 \pm 6.47 \%$ in ELIF and $77.48 \pm 5.98$ in TLIF. The spondylolisthesis rate and reduction rate were similar between two groups $(P>0.05)$.

The average disk space height increased from preoperative $6.85 \pm 2.36 \mathrm{~mm}$ in preoperatively to $11.27 \pm$ $1.29 \mathrm{~mm}$ in postoperatively in ELIF, and from $6.91 \pm 2.54 \mathrm{~mm}$ to $11.42 \pm 1.63 \mathrm{~mm}$ in TLIF. The mean foramen height increased form $13.24 \pm 1.72 \mathrm{~mm}$ in preoperatively to $16.75 \pm 1.17 \mathrm{~mm}$ in postoperatively 
in ELIF, and from $13.41 \pm 1.29 \mathrm{~mm}$ to $17.21 \pm 1.73 \mathrm{~mm}$ in TLIF. The disk height and intervertebral foramen height were better than preoperational $(P<0.01)$, and there were no difference between two groups $(P>$ 0.05).

Table 3 Clinical results date of patients (Means \pm SD)

\begin{tabular}{|c|c|c|c|c|}
\hline Group & ELIF $^{\$}$ & & TLIF & \\
\hline & Preoperative* & Postoperative & Preoperative $\#$ & Postoperative $\#$ \\
\hline VAS & $7.56 \pm 1.13$ & $1.84 \pm 0.89$ & $7.82 \pm 1.09$ & $1.45 \pm 0.91$ \\
\hline ODI & $72.48 \pm 15.63$ & $21.37 \pm 11.82$ & $75.81 \pm 18.49$ & $24.65 \pm 17.36$ \\
\hline Spondylolisthesis (\%) & $30.1 \pm 7.2$ & $5.3 \pm 2.1$ & $31.4 \pm 8.3$ & $5.4 \pm 2.7$ \\
\hline Disk space height (mm) & $6.85 \pm 2.36$ & $11.27 \pm 1.29$ & $6.91 \pm 2.54$ & $11.42 \pm 1.63$ \\
\hline Foramen height(mm) & $13.24 \pm 1.72$ & $16.75 \pm 1.17$ & $13.41 \pm 1.29$ & $17.21 \pm 1.73$ \\
\hline
\end{tabular}

Note: ${ }^{*}$ \#, significant difference $(P<0.01) . \stackrel{\$}{ }$, no significant difference $(P>0.05)$

All patients achieved spinal fusion with no cases of cage extrusion, and no infection, and no cerebrospinal fluid leakage complication. There were three cases of loss of disk space height and foramen height between the initial and final postoperative X-rays suggestive of cage subsidence over time. There were one case of radiculitis (man) at endoscopic group. CT-myelogram revealed the radiculitis patients had normal radiologic findings, and the patient was recovered by neurotrophy drugs and functional exercises after 3 months.

\section{Discussion}

Endoscopic and open procedure of lumbar spine has its advantages and limitations, and the decision about which to perform should mainly be based on the pathologic features of the lumbar.[21-23] Degenerative spondylolisthesis in adults is characterized by the loss of disk height across the affected segment with sagittal translational and is often coupled with rotational deformity.[5] The goal of the surgical treatment of spondylolisthesis was stabilization of the motion segment, decompression of neural elements, reconstitution of disk space height, and restoration of sagittal plane translational and rotational alignment.[24,25] Procedures of endoscopic and open technique share the same goal, the endoscopic can provide a good surgical outcome for decompression with removing the pathogenic structures by minimal invasive procedures.[20] With the data of this cases, the nerve decompression and lumbar interbody fusion reconstruction results were no difference between two methods. However, the patients with endoscopic procedures have less blood loss and faster recovery than open techniques.

Although surgical decompression alone often improves symptoms in the patients with degenerative spondylolisthesis, numerous studies have demonstrated the benefits of concomitant fusion with 
instrumented.[26,27] For spondylolisthesis, the good clinical results not only depend on the sufficient nerve decompression, but also depend on restoration of lumbar lordosis and bone fusion. The ability to reconstruct the lumbar spine after disc evacuation is a radical surgical option due to interbody fusion provide solid fixation of spinal segments while maintaining load-bearing capacity and proper disc height. The original technique of lumbar interbody fusion was modified, transforaminal lumbar interbody fusion technique developed, and the endoscopic lumbar interbody fusion procedures has been adopted.[15,13] With the pedicle screw instrumentation, the endoscopic interbody fusion preserve the load-bearing capacity of the spine, restore the sagittal plane alignment, immediately produces a biomechanically stable postoperative spine, and use the compressive loading on the bone to enhancing the opportunity for arthrodesis.[28,13] The results of this cases show the rate of bone fusion was no difference between two methods, which means the interbody fusion can be accomplished under endoscopic procedures with satisfied results.

Lumbar spine spondylolisthesis presents a significant challenge for spinal surgeon when adopted the endoscopic other than traditional methods, the endoscopic was safe and effective for spondylosis patients as the results of this study. Depending on the severity and clinical features of the spondylolisthesis, it may also be desirable to reduce the forward translation, increase disk space height, decompress the neural elements, and increase or restore lumbar lordosis.[29] TLIF was recommended for spondylolisthesis associated with disc herniation because it is a quick and safe operation resulting in neurological improvement from removed the pathologic tissue and reconstruction the lumbar spine by direction view.[13] TLIF always had the facetectomy produces, and ELIF maintain the facet joint intact by accomplished the nerve decompression and interbody fusion by endoscopic procedures.[30,20] Because perform the endoscopic procedures and percutaneous pedicle screws placement need more time than open surgery, the ELIF procedures had more operation times than TLIF in this series cases.

Endoscopic or open surgery has its superiority, as well as its disadvantages.[8,31] The main shortage of open procedures was the facetectomy, and the main disadvantage of endoscopic need more times to complete the operation at initial cases. The advantage in open surgery was direct view and shot time of operation, and in endoscopic was minimally invasive and maintain the fact joint intact.[32,4] For the block of iliac crest, it is difficult for patients using ELIF surgery in the segments L5-S1, and it is still a thorny problem for these patients using ELIF at now. Severe spondylolisthesis is the most challenging pathology of the lumbar spine to treat surgically because the risks of hemorrhage, and aggravation of neurological deficit are all relatively high, especially for the endoscopic procedures. Then, open surgery may be appropriate as effective and safe procedure treatment for severe spondylolisthesis than endoscopic procedures.

Based on the results of this study, endoscopic lumbar decompression and interbody fusion procedures was effective and safe measure in the treatment of the lumbar spondylolisthesis. Compare to open interbody fusion techniques, endoscopic lumbar interbody fusion was minimally invasive surgery with less bold loss and earlier postoperative recovery. 


\section{Declarations}

\section{Conflict of Interest}

Author Denglu Yan, Zaiheng Zhang, and Zhi Zhang declare that they have no conflict of interest.

\section{Authors' contributions}

DY participated in the design of the study and drafted the manuscript. ZZh participated in the design of the study and coordination and helped to draft the manuscript. ZZ participated in the design of the study and performed the statistical analysis. All authors read and approved the final manuscript.

\section{Ethical approval}

This study was approved by the hospital ethics committee of the First People's Hospital of Zhaoqing, Zhaoqing City, Guangdong Province, and all patients signed had informed consent.

\section{Acknowledgements}

Not applicable.

\section{Author details}

${ }^{1}$ First People's Hospital of Zhaoqing, Zhaoqing, 526000, China

2 People's Hospital of Baoan, Shenzhen, 518101, China

${ }^{3}$ Third Hospital of Guangzhou Medical University, Guangzhou, 510150, China

\section{References}

1. Lee S, Kim SK, Lee SH, Kim WJ, Choi WC, Choi G, Shin SW (2007) Percutaneous endoscopic lumbar discectomy for migrated disc herniation: classification of disc migration and surgical approaches. Eur Spine J 16 (3):431-437. doi:10.1007/s00586-006-0219-4

2. Hsu HT, Chang SJ, Yang SS, Chai CL (2013) Learning curve of full-endoscopic lumbar discectomy. Eur Spine J 22 (4):727-733. doi:10.1007/s00586-012-2540-4

3. Kondo M, Oshima Y, Inoue H, Takano Y, Inanami H, Koga H (2018) Significance and pitfalls of percutaneous endoscopic lumbar discectomy for large central lumbar disc herniation. J Spine Surg 4 (1):79-85. doi:10.21037/jss.2018.03.06

4. Jang JS, An SH, Lee SH (2006) Transforaminal percutaneous endoscopic discectomy in the treatment of foraminal and extraforaminal lumbar disc herniations. J Spinal Disord Tech 19 (5):338343. doi:10.1097/01.bsd.0000204500.14719.2e 
5. Yan DL, Pei FX, Li J, Soo CL (2008) Comparative study of PILF and TLIF treatment in adult degenerative spondylolisthesis. Eur Spine J 17 (10):1311-1316. doi:10.1007/s00586-008-0739-1

6. Chiu JC (2004) Evolving transforaminal endoscopic microdecompression for herniated lumbar discs and spinal stenosis. Surg Technol Int 13:276-286

7. Kuhta M, Bosnjak K, Vengust R (2019) Failure to maintain segmental lordosis during TLIF for onelevel degenerative spondylolisthesis negatively affects clinical outcome 5 years postoperatively: a prospective cohort of 57 patients. Eur Spine J 28 (4):745-750. doi:10.1007/s00586-019-05890-w

8. Tsou PM, Yeung AT (2002) Transforaminal endoscopic decompression for radiculopathy secondary to intracanal noncontained lumbar disc herniations: outcome and technique. Spine J 2 (1):41-48

9. Kolcun JPG, Brusko GD, Basil GW, Epstein R, Wang MY (2019) Endoscopic transforaminal lumbar interbody fusion without general anesthesia: operative and clinical outcomes in 100 consecutive patients with a minimum 1-year follow-up. Neurosurg Focus 46 (4):E14.

doi:10.3171/2018.12.FOCUS18701

10. Ruetten S, Komp M, Godolias G (2005) An extreme lateral access for the surgery of lumbar disc herniations inside the spinal canal using the full-endoscopic uniportal transforaminal approachtechnique and prospective results of 463 patients. Spine (Phila Pa 1976) 30 (22):2570-2578. doi:10.1097/01.brs.0000186327.21435.cc

11. Ruetten S, Komp M, Merk H, Godolias G (2008) Full-endoscopic interlaminar and transforaminal lumbar discectomy versus conventional microsurgical technique: a prospective, randomized, controlled study. Spine (Phila Pa 1976) 33 (9):931-939. doi:10.1097/BRS.0b013e31816c8af7

12. Youn MS, Shin JK, Goh TS, Lee JS (2018) Full endoscopic lumbar interbody fusion (FELIF): technical note. Eur Spine J 27 (8):1949-1955. doi:10.1007/s00586-018-5521-4

13. Hoy K, Bunger C, Niederman B, Helmig P, Hansen ES, Li H, Andersen T (2013) Transforaminal lumbar interbody fusion (TLIF) versus posterolateral instrumented fusion (PLF) in degenerative lumbar disorders: a randomized clinical trial with 2-year follow-up. Eur Spine J 22 (9):2022-2029. doi:10.1007/s00586-013-2760-2

14. Heo DH, Son SK, Eum JH, Park CK (2017) Fully endoscopic lumbar interbody fusion using a percutaneous unilateral biportal endoscopic technique: technical note and preliminary clinical results. Neurosurg Focus 43 (2):E8. doi:10.3171/2017.5.FOCUS17146

15. Schnake KJ (2016) TPLIF (decompression and TLIF) in degenerative spondylolisthesis L4/5. Eur Spine J 25 Suppl 2:272-273. doi:10.1007/s00586-016-4480-x

16. Katzell J (2014) Endoscopic foraminal decompression preceding oblique lateral lumbar interbody fusion to decrease the incidence of post operative dysaesthesia. Int J Spine Surg 8. doi:10.14444/1019

17. Osman SG (2012) Endoscopic transforaminal decompression, interbody fusion, and percutaneous pedicle screw implantation of the lumbar spine: A case series report. Int J Spine Surg 6:157-166. doi:10.1016/j.ijsp.2012.04.001 
18. Zhou Y, Zhang C, Wang J, Chu TW, Li CQ, Zhang ZF, Zheng WJ (2008) Endoscopic transforaminal lumbar decompression, interbody fusion and pedicle screw fixation-a report of 42 cases. Chin $\mathrm{J}$ Traumatol 11 (4):225-231. doi:10.1016/s1008-1275(08)60047-4

19. Niesche M, Juratli TA, Sitoci KH, Neidel J, Daubner D, Schackert G, Leimert M (2014) Percutaneous pedicle screw and rod fixation with TLIF in a series of 14 patients with recurrent lumbar disc herniation. Clin Neurol Neurosurg 124:25-31. doi:10.1016/j.clineuro.2014.06.020

20. Lee SH, Erken HY, Bae J (2017) Percutaneous Transforaminal Endoscopic Lumbar Interbody Fusion: Clinical and Radiological Results of Mean 46-Month Follow-Up. Biomed Res Int 2017:3731983. doi:10.1155/2017/3731983

21. Leu HF, Hauser RK, Schreiber A (1997) Lumbar percutaneous endoscopic interbody fusion. Clin Orthop Relat Res (337):58-63. doi:10.1097/00003086-199704000-00008

22. Polikandriotis JA, Hudak EM, Perry MW (2013) Minimally invasive surgery through endoscopic laminotomy and foraminotomy for the treatment of lumbar spinal stenosis. J Orthop 10 (1):13-16. doi:10.1016/j.jor.2013.01.006

23. Chen Y, Song R, Huang W, Chang Z (2018) Percutaneous endoscopic discectomy in adolescent lumbar disc herniation: a 3- to 5-year study. J Neurosurg Pediatr 23 (2):251-258. doi:10.3171/2018.8.PEDS18442

24. Putzier M, Hartwig T, Hoff EK, Streitparth F, Strube P (2016) Minimally invasive TLIF leads to increased muscle sparing of the multifidus muscle but not the longissimus muscle compared with conventional PLIF-a prospective randomized clinical trial. Spine J 16 (7):811-819. doi:10.1016/j.spinee.2015.07.460

25. Lee HJ, Kim JS, Ryu KS (2016) Minimally Invasive TLIF Using Unilateral Approach and Single Cage at Single Level in Patients over 65. Biomed Res Int 2016:4679865. doi:10.1155/2016/4679865

26. Mehdian H, Kothari M (2017) PLIF and modified TLIF using the PLIF approach. Eur Spine J 26 (Suppl 3):420-422. doi:10.1007/s00586-017-5269-2

27. Mura PP, Costaglioli M, Piredda M, Caboni S, Casula S (2011) TLIF for symptomatic disc degeneration: a retrospective study of 100 patients. Eur Spine J 20 Suppl 1:S57-60. doi:10.1007/s00586-011-1761-2

28. Yang J, Liu C, Hai Y, Yin P, Zhou L, Zhang Y, Pan A, Zhang Y, Zhang L, Ding Y, Xu C (2019) Percutaneous Endoscopic Transforaminal Lumbar Interbody Fusion for the Treatment of Lumbar Spinal Stenosis: Preliminary Report of Seven Cases with 12-Month Follow-Up. Biomed Res Int 2019:3091459. doi:10.1155/2019/3091459

29. Kim CH, Chung CK, Choi Y, Shin S, Kim MJ, Lee J, Park BJ (2015) The Selection of Open or Percutaneous Endoscopic Lumbar Discectomy According to an Age Cut-off Point: Nationwide Cohort Study. Spine (Phila Pa 1976) 40 (19):E1063-1070. doi:10.1097/BRS.0000000000001053

30. Zhou YL, Chen G, Bi DC, Chen X (2018) Short-term clinical efficacy of percutaneous transforaminal endoscopic discectomy in treating young patients with lumbar disc herniation. J Orthop Surg Res 13 (1):61. doi:10.1186/s13018-018-0759-4 
31. Tacconi L, Baldo S, Merci G, Serra G (2018) Transforaminal percutaneous endoscopic lumbar discectomy: outcome and complications in 270 cases. J Neurosurg Sci. doi:10.23736/S03905616.18.04395-3

32. Choi KC, Lee JH, Kim JS, Sabal LA, Lee S, Kim H, Lee SH (2015) Unsuccessful percutaneous endoscopic lumbar discectomy: a single-center experience of 10,228 cases. Neurosurgery 76 (4):372380; discussion 380-371; quiz 381. doi:10.1227/NEU.0000000000000628

\section{Figures}
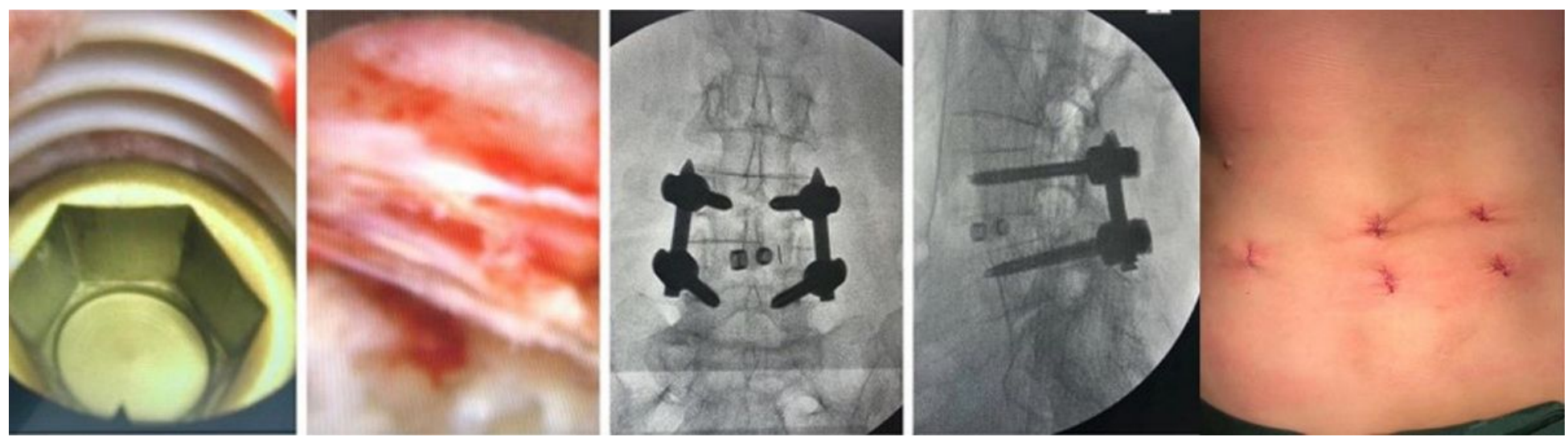

Figure 1

Female patients of 39 years old had the procedures of ELIF for the spondylolisthesis of L4/5. 


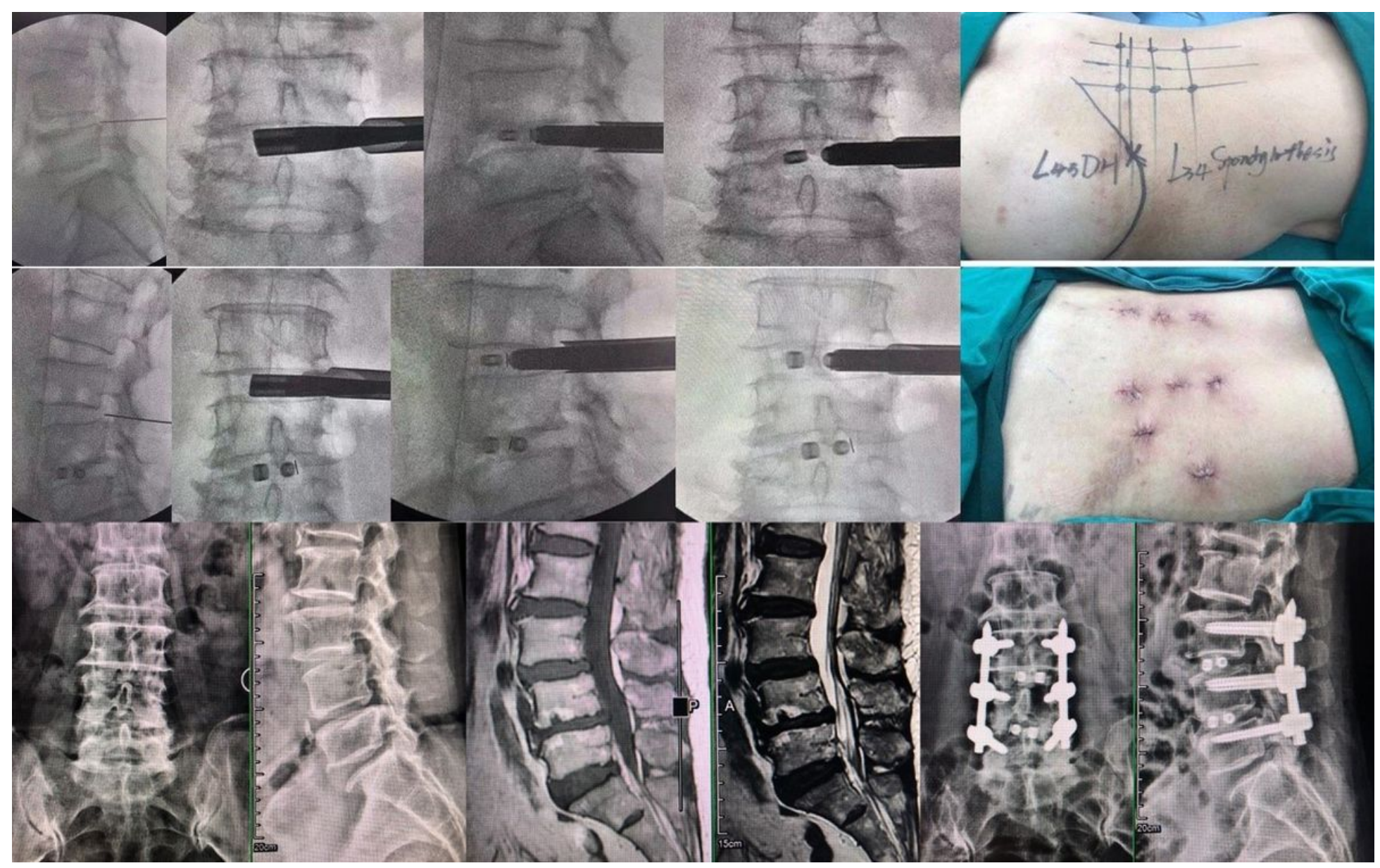

Figure 2

Male patients of 42 years old had the procedures of ELIF for the spondylolisthesis of L3/4 and L4/5 